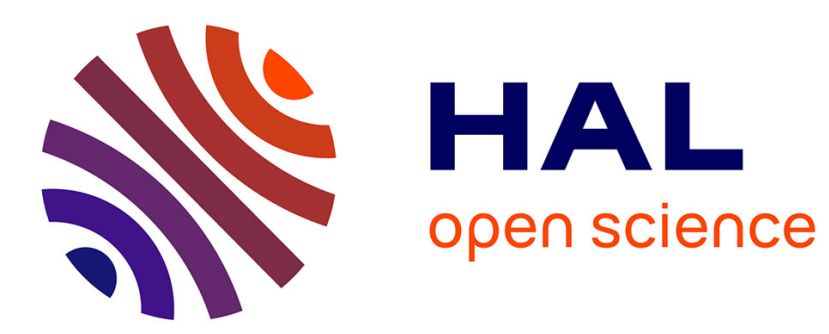

\title{
Measure by interferential conoscopy of the phase distribution acquired from conical diffraction
}

\author{
A Brenier
}

\section{To cite this version:}

A Brenier. Measure by interferential conoscopy of the phase distribution acquired from conical diffraction. Optik, 2021, 246 (21), 10.1016/j.ijleo.2021.167798 . hal-03329243v2

\section{HAL Id: hal-03329243 \\ https://hal.science/hal-03329243v2}

Submitted on 30 Sep 2021

HAL is a multi-disciplinary open access archive for the deposit and dissemination of scientific research documents, whether they are published or not. The documents may come from teaching and research institutions in France or abroad, or from public or private research centers.
L'archive ouverte pluridisciplinaire HAL, est destinée au dépôt et à la diffusion de documents scientifiques de niveau recherche, publiés ou non, émanant des établissements d'enseignement et de recherche français ou étrangers, des laboratoires publics ou privés. 
Optik Volume 246, November 2021, 167798

https://doi.org/10.1016/j.ijleo.2021.167798

\title{
Measure by interferential conoscopy of the phase distribution acquired from conical diffraction
}

\author{
A. Brenier \\ Univ Lyon, Université Claude Bernard Lyon 1, CNRS, Institut Lumière Matière, F-69622, LYON, \\ France
}

Abstract: We have measured the Pancharatman phase difference between the electrical field emerging from the conical diffraction (CD) phenomenon in the $\mathrm{KGd}\left(\mathrm{WO}_{2}\right)_{4}(\mathrm{KGW})$ biaxial crystal and a vertical polarized reference. The full characterisation of the $\widehat{\mathrm{U}}\left(k_{x}, k_{y}\right)$ operator describing the $\mathrm{CD}$ was obtained including polarimetric measurements. We have found that the azimuthal variation of the eigen-values of the dielectric tensor in the theoretical modelling was responsible for an azimuthal variation of the phase of the $\widehat{U}$ operator. The link with the orbital angular momentum of the beam is provided in the cases of a uniformly polarized field and in a vector field.

\section{Introduction}

Generally speaking, the electric field of an electromagnetic wave is a 3-D vector spatially varying both in polarization and phase. This complexity by comparison with a uniformly polarized state has been recognized as the origin of important optical properties with interesting applications. Let us cite the cylindrical vector beams (polarization with a cylindrical symmetry) which can be tighter focused with a high numerical aperture optics [1] and applications in highresolution imaging, surface plasmon excitation, optical trapping, laser machining. Nonseparability of radial and azimuthal polarization can be used to encode information in optical communication [2]. On the other hand, the phase of the electric field is a topic of fundamental interest. It is based on the historical works by S. Panacharatman [3] and M. Berry [4]. The Pancharatman relative phase of two beams can be measured with an interferometric method robust against mechanical and thermal disturbances [5], in addition with polarimetric methods. 
In [6] vectorial fields are created with space-variant gratings and the stability of the vectorial vortices along propagation is studied versus the Pancharatman phase difference with a reference beam. The phase pattern of fields created by spiral phase plates with fractional step height or spatial light modulator have been studied [7-9]. For half-integer phase steps, a chain of additional vortices with alternating charge is observed experimentally and theoretically in the region of low intensity. In these studies the orbital angular momentum (OAM) carried by the beam is associated with the optical vortices with a phase singularity but recently another kind of vortex-free beam carrying OAM received a lot of attention: the elliptical Gaussian beams with a cross-phase. This latter phase (which can be engineered from a spatial light modulator) modifies deeply the propagation and effects such as anisotropic diffraction or spiralling elliptic soliton propagation are observed [10-12].

The field emerging from a biaxial crystal oriented along an optical axis is another spatially varying vector field of interest. Let us recall that this propagation leads to the conical diffraction (CD) of the beam instead of the usual double-refraction and so it emergence as a hollow cylinder near the focal plane $[13,14]$. Nowadays numerous applications of CD have been found [15], among them let us only cite the optical trapping of light, the polarization multiplexing for optical communications, the super resolution microscopy. The key-point for the present work is that the electric field emerges with a space-variant polarization and phase. Berry [16] described this field with two components: $\mathrm{B}_{0}$ one with no azimuthal dependence of the phase and $\mathrm{B}_{1}$ component with an azimuthal phase at the origin of the OAM of the wave. In [17], the $\mathrm{B}_{1}$ component was selected with a quarter-wave plate and a rotated linear polarizer and the intensities of the resulting linear polarized beams with fractional OAM between 0 and 1 $\hbar /$ photon were visualized with a camera. The interferograms produced by the interference with a linear polarized reference beam are shown and reveal the OAM. However, an interferogram itself is not the value of a Pancharatman phase difference in the meaning of Ref. [5-9], and so the phase distribution of the field emerging from $C D$ is not exhibited. The purpose of the study in Ref. [18] is the rings located in the focal plane of a focused Gaussian beam. On the ring the polarization is linear with an azimuthal varying orientation and the authors demonstrate with an interferometric method that this $\mathrm{CD}$ output linear polarization is equivalent to the one produced with a (rotated) linear polarizer. In this case the output state is the projection $|p><p|$ of the input state on the polarizer direction of transmission ( $\mid$ out $>=|p><p|$ in $>$ ) and so of course the input and output states present no Pancharatman phase difference. This peculiar situation does not occur generally in the CD phenomenon. 
The present work is devoted to measure the Pancharatman phase difference between the electrical field emerging from the $\mathrm{CD}$ phenomenon in the $\mathrm{KGd}\left(\mathrm{WO}_{2}\right)_{4}(\mathrm{KGW})$ biaxial crystal and a vertical polarized reference. It is shown that the azimuthal variation of the eigen-values of the dielectric tensor is necessary in the theoretical modelling to get agreement with the experimental data. The KGW crystal was chosen because it is a "textbook case" for the CD phenomenon and this prevailing position highlights the fact that its study was not exhausted, the phases accompanying the $\mathrm{CD}$ being not investigated in accordance with their importance. The link with the orbital angular momentum is provided.

\section{The vectorial field generated from $\mathrm{CD}$}

The electric field $\mathbf{E}$ of a monochromatic input wave at the entrance face of the biaxial can be decomposed in plane waves from its $2 \mathrm{D}$-Fourier transform (FT) $\hat{\mathbf{E}}\left(0, k_{x}, k_{y}\right), k_{x}, k_{y}$ being the transverse components of the wave-vector in the xyz crystal frame ( $\mathrm{z}$ is the optical axis direction). At the output face of the crystal with thickness $L$ the 2D-polarization is a vectorial field $\left[\hat{\mathbf{E}}\left(L, k_{x}, k_{y}\right)\right]_{x y}$ obtained from the unitary operator representing a retarder which introduces a phase $\mu$ between the two eigen-modes, the latter being described by the two $\boldsymbol{r}_{\mathbf{0}}$ $(\mathrm{x}, \mathrm{y}, \mathrm{z})$ and $-\boldsymbol{r}_{\mathbf{0}}$ vectors on the Poincaré sphere [19] (a circular basis is used and $\mathrm{x}, \mathrm{y}, \mathrm{z}$ are the Stokes parameters of the eigen-mode):

$$
\widehat{\mathrm{U}}\left(k_{x}, k_{y}\right)=e^{i \varnothing}\left[\begin{array}{cc}
\cos \left(\frac{\mu}{2}\right) & -i \sin \left(\frac{\mu}{2}\right)(x-i y) \\
-i \sin \left(\frac{\mu}{2}\right)(x+i y) & \cos \left(\frac{\mu}{2}\right)
\end{array}\right]
$$

where $\boldsymbol{r}_{\mathbf{0}}$ has been restricted to the equatorial plane $\mathrm{z}=0$ because the crystal in this work is only birefringent and $\varnothing$ is a general phase (all the parameters are $k_{x}, k_{y}$ dependent).

On the other hand, let us write as $e^{i \Phi}\left[\begin{array}{c}\cos \left(\frac{\theta}{2}\right) \\ \sin \left(\frac{\theta}{2}\right) e^{i \varphi}\end{array}\right]$ the Jones vector of the output polarization (characterized by its polar and azimuthal angle $\theta$ and $\varphi$ on the Poincaré sphere) obtained launching a left-circular (LC) polarization $\left[\begin{array}{l}1 \\ 0\end{array}\right]$. The output polarization can also be obtained by the product $\widehat{\mathrm{U}}\left[\begin{array}{l}1 \\ 0\end{array}\right]$. Then the left column of the $\widehat{\mathrm{U}}\left(k_{x}, k_{y}\right)$ operator in Eq. (1) can be identified accordingly with the Jones vector. Launching a right-circular (RC) polarization $\left[\begin{array}{l}0 \\ 1\end{array}\right]$ in Eq. (1), the second column of the $\widehat{\mathrm{U}}\left(k_{x}, k_{y}\right)$ operator can also be identified. The full identification is: 


$$
\widehat{\mathrm{U}}\left(k_{x}, k_{y}\right)=e^{i \Phi}\left[\begin{array}{cc}
\cos \left(\frac{\theta}{2}\right) & -\sin \left(\frac{\theta}{2}\right) e^{-i \varphi} \\
\sin \left(\frac{\theta}{2}\right) e^{i \varphi} & \cos \left(\frac{\theta}{2}\right)
\end{array}\right]
$$

where all the parameters are $k_{x}, k_{y}$ dependent.

The experimental determination of the $\theta\left(k_{x}, k_{y}\right)$ and $\varphi\left(k_{x}, k_{y}\right)$ angular distributions via a polarimetric method is explained in section 4.1 . Their theoretical determination can be explicated summarizing the origin of Eq. (1) [20- 23]. First, the dielectric tensor representing the birefringence of the biaxial crystal, which is diagonal in the principal frame, is expressed in the transverse frame $\mathrm{x}_{1}, \mathrm{x}_{2}, \mathrm{x}_{3}$ (whose third axis $\mathrm{x}_{3}$ is parallel to the refracted wave-vector) with two rotations (one for the transition principal frame $\rightarrow x y z$ frame, one for $x y z \rightarrow$ transverse frame). Then it is possible to calculate the components in the transverse frame of the two eigen_modes $( \pm)$, the two eigen-values $k_{ \pm}$and the transfer matrix from the transverse frame towards the eigen-modes. The electric field inside the crystal has components on the two eigenmodes propagating as $\exp \left[i\left(k_{x} x+k_{y} y+k_{z \pm} z\right)\right]$, with:

$$
k_{z \pm}=\sqrt{k_{ \pm}^{2}-\left(k_{x}^{2}+k_{y}^{2}\right)}
$$

The Û operator is:

$$
\widehat{\mathrm{U}}=\exp \left\{i d P\left[\begin{array}{cc}
k_{z+} & 0 \\
0 & k_{z-}
\end{array}\right] P^{-1}\right\}
$$

where $P$ is the transfer matrix from the xy-frame towards the eigen-modes frame, $d$ is the crystal thickness.

The parabolic approximation leads to smart results for the eigen-values and the eigen-modes of the dielectric tensor, and consequently for the operator $\widehat{U}[20,21]$. In the present work we used the exact refractive indices and eigen-modes obtained by diagonalizing the dielectric tensor, as it is detailed in Ref. [22, 23] (including birefringence and possibly bi-anisotropy), and so we obtained numerically with the Matlab package the $\widehat{U}$ operator and the $\theta\left(k_{x}, k_{y}\right)$ and $\varphi\left(k_{x}, k_{y}\right)$ angular distributions presented in section 4.1 .

\section{Experimental set-up for interferential conoscopy}

\subsection{General description}


We built an interferential conoscope (Fig. 1) which is basically constituted with two arms of the same length. A beam from a $1064 \mathrm{~nm}$ YAG:Nd laser with its linear polarization at $45^{\circ}$ is separated by the PBS253 (Thorlabs) polarizing beam-splitter in a vertical polarized beam (arm $1=\mathrm{PBS} 253+\mathrm{M} 1+\mathrm{BS})$ and a horizontal polarized beam (arm 2=PBS253+M2+BS). In arm 1 a liquid crystal retarder LCC1223-B (Thorlabs) is installed while in arm 2 a quarter-wave plate produces a LC polarization.

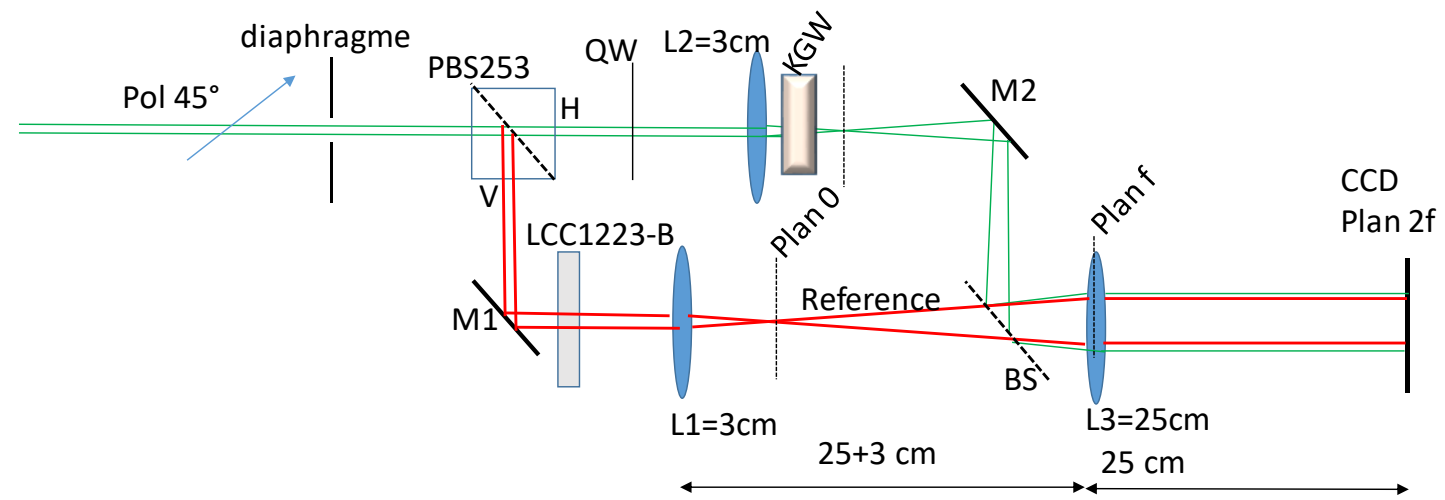

Fig 1. Set-up for interferentiel conoscopy. After the BS the two beams have been separated for a better visualization by actually they are superimposed.

In each arm two identical doublets (AC254-30 from Thorlabs, focal length $=3 \mathrm{~cm}$ ) are installed at the same distance than PBS253. The important point of the set-up is that the length L1-BS is tuned as close as possible than the length L2-M2-BS. So we obtained two divergent beams, identical (at this point the KGW crystal in arm 2 is not installed) and superimposed after the BS.

\subsection{Record of the FT field located at plan 0 by the camera}

In each arm (Fig. 1) the plan of interest under study is the "plan 0". Let us show that the FT of the electric field in this plane is recorded by the camera with the help of a new L3 lens. At the output of BS, a third doublet $\mathrm{L}_{3}$ (AC254-250 from Thorlabs, focal length $=\mathrm{f}=25 \mathrm{~cm}$ ) is installed at the distance f from plan 0 , the camera being in the plan $2 \mathrm{f}$. L3 realizes the FT of each beam 1 or 2 at plan 0 . More precisely, in the transverse plane, $\mathrm{L}_{3}$ adds a quadratic phase to the electric field, that is to say $\mathrm{L}_{3}$ multiplies the field by a phase factor whose FT can be taken as:

$$
\widehat{L}\left(k_{x}, k_{y}\right)=\frac{-2 \pi i f}{k} \exp \left(i \frac{k_{x}^{2}+k_{y}^{2}}{2 k} f\right)
$$


So if $\widehat{\varepsilon_{f-}}\left(k_{x}, k_{y}\right)$ is the FT field immediately before the $\mathrm{L}_{3}$ lens at $\mathrm{z}=\mathrm{f}$, immediately after the lens the FT field will be:

$$
\widehat{\varepsilon_{f+}}\left(k_{x}, k_{y}\right)=\widehat{L \varepsilon_{f-}}=\left(\frac{1}{2 \pi}\right)^{2} \widehat{L} * \widehat{\varepsilon_{f-}}
$$

where * represents the convolution product. Taking into account that a separation by a distance $\Delta z$ between two planes introduces the phase factor in the Fourier space $\exp \left(i k \Delta z-i \frac{k_{x}^{2}+k_{y}^{2}}{2 k} \Delta z\right)$, using $\Delta z=f$ before and after $\mathrm{L}_{3}$, the TF field recorded by the $\mathrm{CCD}$ at position $\mathrm{z}=2 \mathrm{f}$ is obtained from the one at $\mathrm{z}=0$ with the formula:

$$
\widehat{\varepsilon_{2 f}}\left(k_{x}, k_{y}\right)=\frac{-2 \pi i f}{k} \exp (2 i k f) \varepsilon_{0}\left(-k_{x} \frac{f}{k},-k_{y} \frac{f}{k}\right)
$$

Each pixel of the CCD camera, located in the L3 focal plane with (X, Y) transverse spatial coordinates, measures an electric field given by the inverse FT of Eq. (7). The expected result is:

$$
\varepsilon_{2 f}(X, Y)=\frac{-i}{2 \pi} \frac{k}{f} \exp (2 i k f) \widehat{\varepsilon_{0}}\left(k_{x}=X \frac{k}{f}, k_{y}=Y \frac{k}{f}\right)
$$

showing that the camera records directly the wanted FT field in the plane 0. This formula will be applied to arm 2 launched alone (field under study) for its polarization determination (section 4.1) or both arm 1 and arm 2 launched (interferences of the two beams) for Pancharatman phase determination in section 3.3. So we need to calculate $\widehat{\varepsilon_{0}}$ for each field.

In our experimental set up the reference beam is Gaussian, focused in the plan 0 and is vertically polarized, so in arm 1:

$$
\widehat{\varepsilon_{0}}=e^{i \emptyset_{P}} \mid \widehat{r e f}>=\sqrt{I_{\text {ref }}} e^{i \emptyset_{P}} \exp \left(-\frac{w_{0}^{2}\left(k_{x}^{2}+k_{y}^{2}\right)}{4}\right) \frac{1}{\sqrt{2}}\left[\begin{array}{r}
1 \\
-1
\end{array}\right]
$$

where the beam waist is $w_{0}=17 \mu m$. Let us notice that in Eq. (9) we have isolated the phase $\emptyset_{P}$ produced by the liquid crystal retarder.

Except its polarization, the beam in arm 2 is identical to $\mid \widehat{r e f}>$ in absence of the biaxial crystal. The $\mathrm{CD}$ is obtained from a $\mathrm{KGd}\left(\mathrm{WO}_{2}\right)_{4}(\mathrm{KGW})$ crystal (with thickness $d=0.315 \mathrm{~cm}$ ) installed slightly before the plane 0 at abscissa $-z_{c}$. In the plane 0 , using the $\widehat{U}$ operator (2) acting on a LC polarization $\left[\begin{array}{l}1 \\ 0\end{array}\right]$, the TF of the field is: 


$$
\widehat{\varepsilon_{0}}=\mid \widehat{E}\left(k_{x}, k_{y}\right)>=\sqrt{I_{E}} \exp \left(-\frac{w_{0}^{2}\left(k_{x}^{2}+k_{y}^{2}\right)}{4}\right) \exp \left(i \frac{k_{x}^{2}+k_{y}^{2}}{2 k} d\right) e^{i \Phi}\left[\begin{array}{c}
\cos \left(\frac{\theta}{2}\right) \\
\sin \left(\frac{\theta}{2}\right) e^{i \varphi}
\end{array}\right]
$$

To obtain the phase factor in Eq. (10) we have used the evolution of the wave as $\exp \left(-i k_{z} z_{c}\right)$ at the crystal entrance face with abscissa $-z_{c}, \exp \left(-i k_{z} z_{c}\right) \widehat{\mathrm{U}}$ at the crystal exit face with abscissa $-z_{c}+d$ and $\exp \left(-i k_{z} z_{c}\right) \widehat{U} \exp \left(i k_{z}\left(0-\left(-z_{c}+d\right)\right)\right.$ at abscissa 0 where the

Gaussian beam is focused. $k_{z}=k-\frac{k_{x}^{2}+k_{y}^{2}}{2 k}$ in air and we have dropped the non useful term $\exp (i k d)$

\subsection{Pancharatman phase difference between the reference and the field}

Using Eq. (8), (9) and (10) the intensity of the interference of the two fields recorded by the $\mathrm{CCD}$ is:

$$
\begin{aligned}
& I\left(k_{x}, k_{y}\right)=\left|e^{i \emptyset_{P}}\right| \widehat{r e f}\left(k_{x}, k_{y}\right)>+\left|\widehat{E}\left(k_{x}, k_{y}\right)>\right|^{2} \\
& I\left(k_{x}, k_{y}\right)=I_{\text {ref }}+I_{E}+2|<\widehat{r e f}| \widehat{E}>\mid \cos \left(\emptyset_{P}-\arg <\widehat{r e f} \mid \widehat{E}>\right)
\end{aligned}
$$

The Pancharatman phase difference between the reference and the field under study is the phase retardation introduced by the liquid crystal retarder which maximizes $I\left(k_{x}, k_{y}\right)$ for each camera pixel, that is to say:

$$
\emptyset_{P}\left(k_{x}, k_{y}\right)=\arg <\widehat{r e f}\left(k_{x}, k_{y}\right) \mid \hat{E}\left(k_{x}, k_{y}\right)>
$$

The key-point is that the two arms are identical, implying that the two beams evolve in the two arms with the part of the phase which is quadratic (originating from the Gaussian evolution) differs only by a calculable $\frac{k_{x}^{2}+k_{y}^{2}}{2 k} d$ phase factor (Eq. (9) compared with Eq. (10)). Otherwise unwanted supplementary quadratic phase factors should be included in the scalar product in Eq. (12), making more difficult the analysis.

The Pancharatman phase $\emptyset_{P}$ produced by the liquid crystal retarder was increased from 0 to 2 $\pi$ in up to 25 steps. For a few of them we present in Fig. (2) typical interference patterns visualizing Eq. (11) and corresponding to the fields $\mid \widehat{E}\left(k_{x}, k_{y}\right)>$ involved in the 4.2, 4.3.2 and 4.3.3 following subsections. 

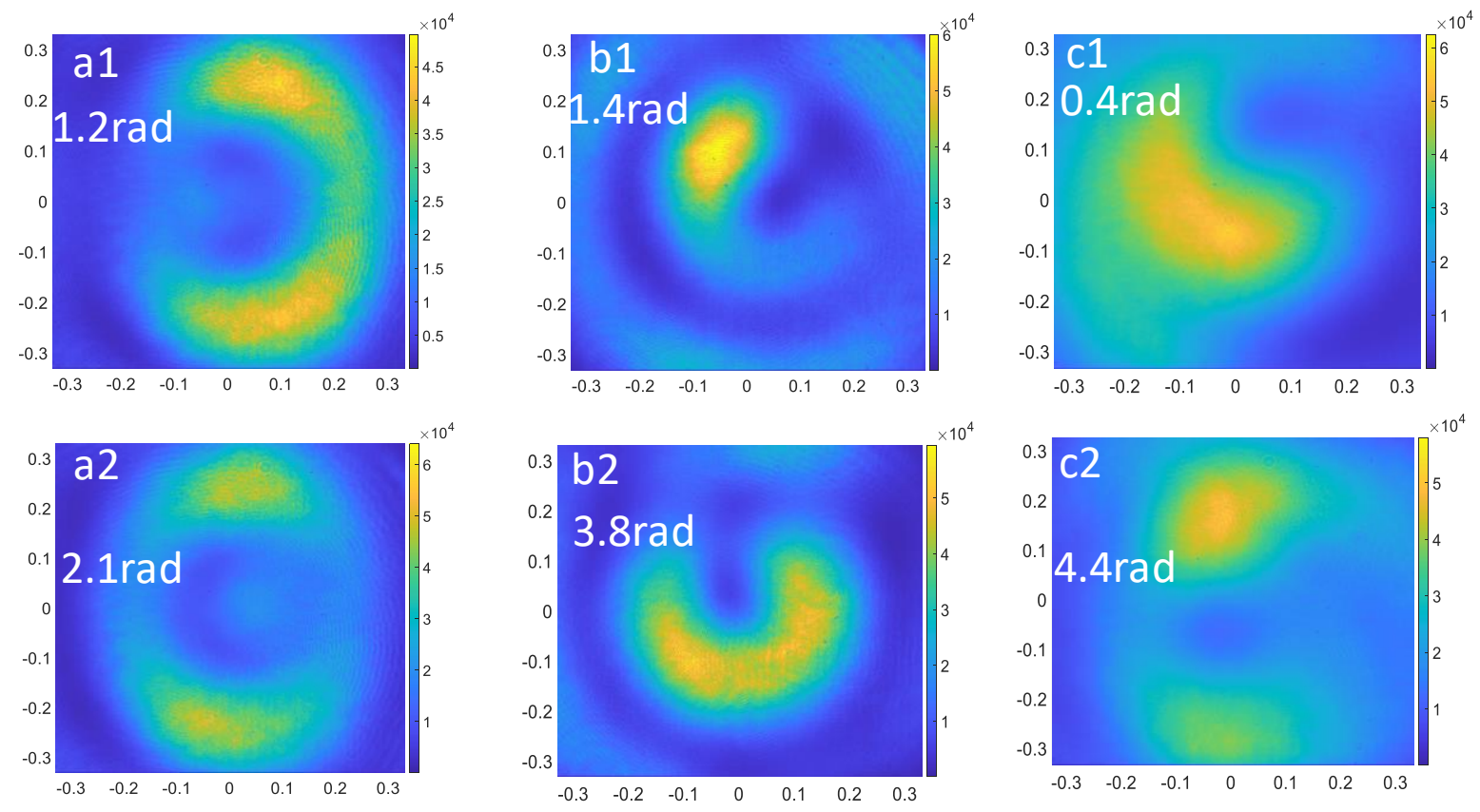

Fig. 2 Typical interferograms visualizing Eq. (7), the values in white are $\emptyset_{P}$. a1-a2, b1-b2, c1c2 correspond respectively to the fields of subsections 4.2, 4.3.2 and 4.3.3 .

\section{Experimental results and interpretation}

\subsection{Polarization of the FT electric field after CD}

The polarization of the CD electric field in different planes of free-space is known [24]. However, for the purpose of the present work we need to measure it in the Fourier space in the specific conditions of the conoscopy experimental set-up of Fig. 1 (the reference beam is stopped in this subsection). This is a necessary step to determine the Û operator in Eq. (2). The $\theta\left(k_{x}, k_{y}\right)$ and $\varphi\left(k_{x}, k_{y}\right)$ angular positions of the polarization on the Poincare sphere are related to the $S_{1}, S_{2}$ and $S_{3}$ Stoke parameters:

$$
\begin{gathered}
\theta=\operatorname{acos}\left(S_{3} / I\right) \\
\varphi=\arg \left(S_{1}+i S_{2}\right)
\end{gathered}
$$

where $I\left(k_{x}, k_{y}\right)$ is the intensity recorded by the camera of the output beam after launching a LC beam in arm 2 alone and:

$$
\begin{array}{r}
S_{1}=I\left(0^{\circ}\right)-I\left(90^{\circ}\right) \\
S_{2}=I\left(45^{\circ}\right)-I\left(135^{\circ}\right)
\end{array}
$$




$$
S_{3}=I(L C)-I(R C)
$$

where $I\left(0^{\circ}\right), I\left(90^{\circ}\right), I\left(45^{\circ}\right), I\left(135^{\circ}\right), I(L C)$ and $I(R C)$ are respectively the intensities recorded by the camera through a linear polarizer horizontal, vertical, at $45^{\circ}$, at $135^{\circ}$, and through a LC and a RC polarizers [14], all these optical elements being located in the path $\mathrm{L}_{3}-\mathrm{CCD}$ in Fig. 1.
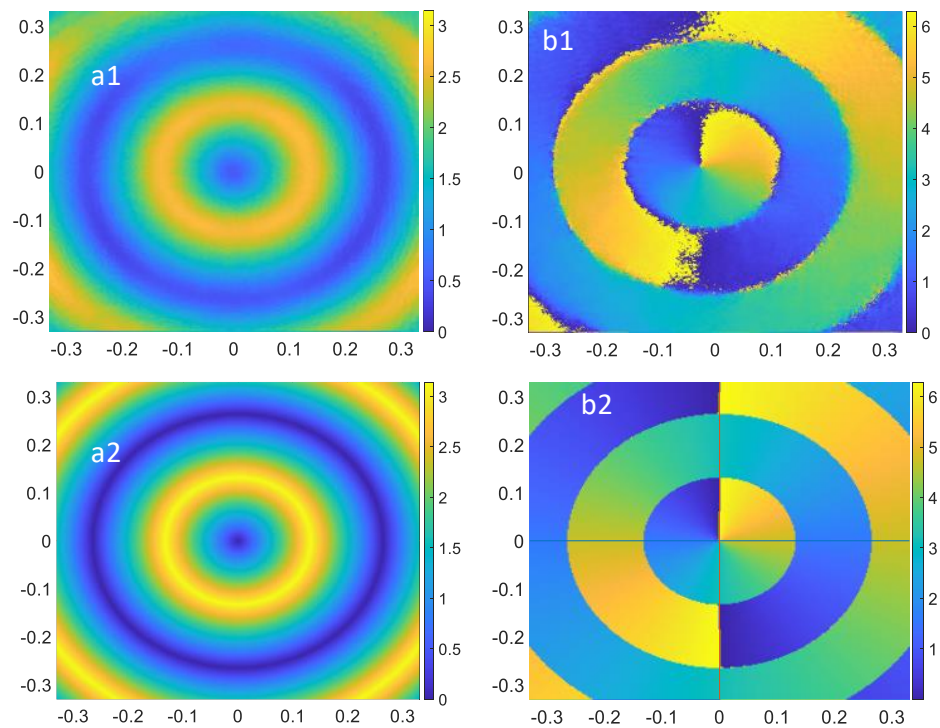

Fig. 3: $\theta\left(k_{x}, k_{y}\right)(\mathrm{a} 1)$ : experimental,(a2): theoretical) and $\varphi\left(k_{x}, k_{y}\right)(\mathrm{b} 1)$ : experimental,(b2): theoretical) angular positions of the polarization on the Poincare sphere.

The $\theta\left(k_{x}, k_{y}\right)$ and $\varphi\left(k_{x}, k_{y}\right)$ angular distributions obtained experimentally are represented in Fig. 3 (a1) and (b1) respectively. The calculated distributions (Fig. 3 (a2) and (b2) are in agreement despite some noise in the experimental data.

\subsection{Phase of the FT electric field after CD}

The phase $\Phi\left(k_{x}, k_{y}\right)$ of the FT electric field after CD in Eq. (2) can be measured by locating in the path $\mathrm{L}_{3}-\mathrm{CCD}$ in Fig. 1 a quarter-wave plate with its slow axis at $45^{\circ}$ from horizontal, followed by a vertical linear polarizer. The full Jones matrix of both these two elements is $\frac{1}{\sqrt{2}}\left[\begin{array}{cc}1 & 0 \\ -1 & 0\end{array}\right]$, and this operator modifies the FT field of Eq. (10) as:

$$
\mid \widehat{E}^{\prime}\left(k_{x}, k_{y}\right)>=\exp \left(i \frac{k_{x}^{2}+k_{y}^{2}}{2 k} d\right) e^{i \Phi}\left[\begin{array}{c}
1 \\
-1
\end{array}\right]
$$

where we have dropped all the real positive factors and kept only the phase factors of interest. With the same treatment the reference beam (Eq. (9)) remains of course vertically polarized $\left[\begin{array}{c}1 \\ -1\end{array}\right]$. The Pancharatman phase from Eq. (12) is obtained as: 


$$
\emptyset_{P}\left(k_{x}, k_{y}\right)=\Phi\left(k_{x}, k_{y}\right)+\frac{k_{x}^{2}+k_{y}^{2}}{2 k} d
$$

$\emptyset_{P}\left(k_{x}, k_{y}\right)$ being measured by the camera of the interferential conoscope, the experimental phase function $\Phi\left(k_{x}, k_{y}\right)$ is extracted and represented in Fig. 4 (a1). It can be compared with its theoretical calculation Fig. 4 (b1).
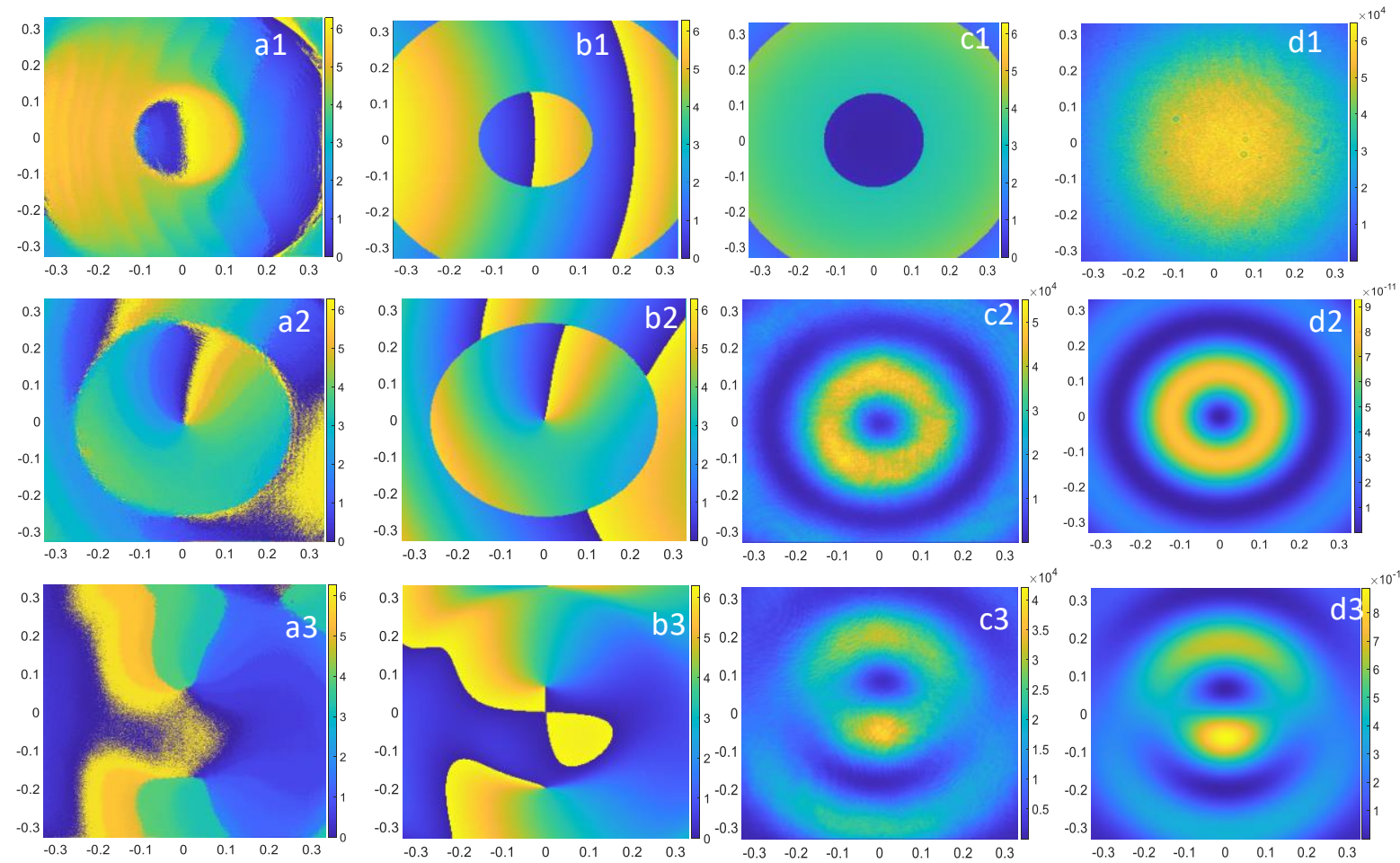

Fig. 4 a1, b1, c1: phase of the $\widehat{U}$ operator, d1: output intensity after CD; a2, b2: experimental and theoretical Pancharatman phase difference between the reference beam and the charge 1 beam produced by $\mathrm{CD} ; \mathrm{c} 2, \mathrm{~d} 2$ : experimental and theoretical intensity of the charge 1 beam produced by CD; a3, b3: experimental and theoretical Pancharatman phase difference between the reference beam and the fractional charge beam produced by $\mathrm{CD} ; \mathrm{c} 3, \mathrm{~d} 3$ : experimental and theoretical intensity of the fractional charge beam produced by $\mathrm{CD}$.

We can observe that the $\left(k_{x}, k_{y}\right)$ dependence of $\Phi$ is not axially symmetric. This is the consequence of the azimuthal variation of the eigen-values of the dielectric tensor, and this behaviour cannot be explained with well-used approximations such as the one in Ref. [21] which produce the phase distribution represented in Fig. 4 (c1).

To complete the characterization of the the $\widehat{U}$ operator we added the intensity of the beam after CD, launching a Gaussian beam (Fig. 4 (d1)). 


\subsection{Application to the OAM determination}

\subsubsection{Link with the OAM}

This section is motivated because the electromagnetic wave emerging after CD carries an OAM [25] due to the missing of azimuthal symmetry around the optical axis. The evaluation of the z-component of the OAM of a $\left|\widehat{E}>=\widehat{E}_{V}\right| \widehat{V}>+\widehat{E}_{H} \mid \widehat{H}>$ field in the Fourier space (decomposed in vertical and horizontal components) can be performed with Darwin result [26], restricted here to the $2 \mathrm{D}$ monochromatic case. Writing the OAM operator as $\hat{L}_{z}=-i \hbar \frac{\partial}{\partial \phi}(\phi$ is the azimuthal angle in Fourier space), the OAM ( $\hbar /$ photon) is:

$$
l_{z}=\frac{\mathcal{R}_{e} \iint\left[{\widehat{E_{V}}}^{*}\left(\widehat{L}_{z} \widehat{E_{V}}\right)+{\widehat{E_{H}}}^{*}\left(\widehat{L}_{z} \widehat{E_{H}}\right)\right] d k_{x} d k_{y}}{\iint\left[\left.\left|{\widehat{E_{V}}}^{2}+\right| \widehat{E_{H}}\right|^{2}\right] d k_{x} d k_{y}}
$$

where $\mathcal{R}_{e}$ means "real part". Let us recall that the OAM can be determined by measuring the intensity distribution with a camera located in the focal plane of a cylindrical lens in two orientations [27], but in this work we follow another route.

Writing $\hat{E}_{V}=\langle V| \hat{E}>=\sqrt{I_{V}} e^{i \emptyset_{P V}}$, the contribution to $l_{z}$ of the $\mathrm{V}$-component is reduces to $I_{V} \partial_{\phi} \emptyset_{P V}$ where $\emptyset_{P V}$ is the Pancharatman phase difference between the field and a vertical polarized reference. The full OAM including a horizontal polarized contribution is:

$$
l_{z}=\frac{\iint\left(I_{V} \partial_{\phi} \emptyset_{P V}+I_{H} \partial_{\phi} \emptyset_{P H}\right) d k_{x} d k_{y}}{\iint\left(I_{V}+I_{H}\right) d k_{x} d k_{y}}
$$

\subsubsection{Application to a uniformly polarized field}

We choose as an example the linear polarized field provided by locating in the path $\mathrm{L}_{3}-\mathrm{CCD}$ in Fig. 1 a quarter-wave plate with its slow axis at $-45^{\circ}$ from horizontal followed by a vertical linear polarizer. The full Jones matrix of both these two elements is $\frac{1}{\sqrt{2}}\left[\begin{array}{cc}0 & -1 \\ 0 & 1\end{array}\right]$, and this operator modifies the FT field of Eq. (10) as:

$$
\mid \widehat{E}^{\prime}\left(k_{x}, k_{y}\right)>=\exp \left(i \frac{k_{x}^{2}+k_{y}^{2}}{2 k} d\right) e^{i(\Phi+\varphi)} \frac{1}{\sqrt{2}} \sin \left(\frac{\theta}{2}\right)\left[\begin{array}{c}
1 \\
-1
\end{array}\right]
$$

while the reference beam (Eq. (9)) remains of course vertically polarized $\left[\begin{array}{c}1 \\ -1\end{array}\right]$. The Pancharatman phase from Eq. (12) is obtained as:

$$
\emptyset_{P}\left(k_{x}, k_{y}\right)=\Phi\left(k_{x}, k_{y}\right)+\varphi\left(k_{x}, k_{y}\right)+\pi+\frac{k_{x}^{2}+k_{y}^{2}}{2 k} d
$$


The $\emptyset_{P}\left(k_{x}, k_{y}\right)$ phase is measured by the camera of the interferential conoscope (Fig. 4(a2)). It can be compared with its theoretical calculation Fig. 4 (b2).

Let us calculate the OAM with Eq. (19) in which $I_{H}=0$ and the $I_{V}\left(k_{x}, k_{y}\right)$ intensity measured experimentally in Fig. 4 (c2) and calculated in Fig. 4 (d2) has no azimuthal $\phi$-dependence. We find from Fig. 4 (b1-b2) that the azimuthal variation of $\emptyset_{P}\left(k_{x}, k_{y}\right)$ in polar coordinates is $2 \pi$, which leads to OAM=1. Contrarily to Fig 4(a1), a phase vortex coincident with the nil field intensity is located in the centre.

\subsubsection{Application to a vector field}

We study in this sub-section the CD field emerging from the crystal without any optical elements in the path $\mathrm{L}_{3}$-CCD in Fig. 1. The FT of this field is given by Eq. (10) while the reference field is still vertical polarized (Eq. (9)). Then the Pancharatman phase difference between these two fields is provided by Eq. (12) as:

$$
\emptyset_{P}\left(k_{x}, k_{y}\right)=\Phi\left(k_{x}, k_{y}\right)+\arg \left(\cos \left(\frac{\theta}{2}\right)-\sin \left(\frac{\theta}{2}\right) e^{i \varphi}\right)+\frac{k_{x}^{2}+k_{y}^{2}}{2 k} d
$$

This experimental phase is represented in Fig. 4 (a3) and can be compared with the theoretical calculation Fig. 4 (b3). As in subsection 4.2 the azimuthal variation of the eigen-values of the dielectric tensor is necessary to get an agreement of the experimental and theoretical phases. Let us notice the noise $0 / 2 \pi$ (blue/yellow) in the experimental figure.

Let us calculate the OAM with Eq. (19) adding the two vertical and horizontal contributions. The $I_{V}\left(k_{x}, k_{y}\right)$ intensity was measured experimentally (Fig. 4 (c3), see sub-section 4.1) and calculated in Fig. 4 (d3) (and similarly but not shown for $I_{H}\left(k_{x}, k_{y}\right)$ ).We find from Fig. 4 (a3b3) that the azimuthal variation of $\emptyset_{P}\left(k_{x}, k_{y}\right)$ leads to $\mathrm{OAM}=0.267$ for the vertical contribution and 0.249 for the horizontal. The full OAM is then the fraction 0.516 , the two phase vortices are coincident with the two nil field intensities.

\section{Conclusion}

We have measured the Pancharatman phase difference between the electrical field emerging from the $\mathrm{CD}$ phenomenon in the $\mathrm{KGd}\left(\mathrm{WO}_{2}\right)_{4}(\mathrm{KGW})$ biaxial crystal and a vertical polarized reference. The full characterisation of the $\widehat{\mathrm{U}}\left(k_{x}, k_{y}\right)$ operator describing the $\mathrm{CD}$ was obtained. We have found that the azimuthal variation of the eigen-values of the dielectric tensor in the theoretical modelling was responsible for an azimuthal variation of the phase of the $\widehat{U}\left(k_{x}, k_{y}\right)$ 
operator. The link with the orbital angular momentum of the beam is provided in the cases of a uniformely polarized field and in a vector field.

\section{References}

1. Q. Zhan, "Cylindrical vector beams: from mathematical concepts to applications", Advances in Optics and Photonics 1, 1-57 (2009).

2. G. Milione,T. A. Nguyen,J. Leach, D. A. Nolan, and R. R. Alfano, "Using the nonseparability of vector beams to encode information for optical communication", Opt. Lett. 40 (21), 4887 (2015).

3. S. Pancharatman, "Generalized theory of interference, and it, s applications", Proc. Indian Acad. Sci. A 44, 247-262 (1956).

4. M.V. Berry, "The Adiabatic Phase and Pancharatnam's Phase for Polarized Light", Journal of Modern Optics, 34:11, 1401-1407 (1987).

5. J. C. Loredo, O. Ortíz, R. Weingärtner and F. De Zela, "Measurement of Pancharatnam's phase by robust interferometric and polarimetric methods", Phys. Rev. A 80, 012113 (2009).

6. A. Niv, G. Biener, V. Kleiner, E. Hasman, "Manipulation of the Pancharatnam phase in vectorial vortices”, Opt. Exp. 14 (10), 4208 (2006).

7. M. V. Berry, "Optical vortices evolving from helicoidal integer and fractional phase steps", J. opt. A: pure Appl. Opt. 6, 259-268 (2004).

8. J. Leach, E. Yao and M. J. Padgett, "Observation of the vortex structure of a non-integer vortex beam", New J. Phys., 6, 71 (2004).

9. J. B. Götte, S. Franke-Arnold, R. Zambrini, and S. M. Barnett, "Quantum formulation of fractional orbital angular momentum”, J. Mod. Opt., 54 (12), 1723-1738 (2007).

10. G. Liang and Q. Wang, "Controllable conversion between Hermite Gaussian and Laguerre Gaussian modes due to cross phase”, Opt. Express, 27 (8), 10684 (2019).

11. G. Liang, "Revolving and spinning of optical patterns by two coaxial spiraling elliptic beams in nonlocal nonlinear media”, Opt. Express, 27 (10), 14667 (2019).

12. G. Liang, H. Zhang, L.Fang, Q. Shou, W. Hu and Q. Guo, "Influence of transverse cross-phases on propagation of optical beams in linear and nonlinear regimes", Laser Photonics Rev., 14, 2000141 (2020). 
13. L. Landau and E. Lifchitz, Electrodynamique des milieux continus, Ed. Mir Moscou 1969.

14. M. Born, E. Wolf, A. B. Bhatia, Principles of optics: electromagnetic theory of propagation, interference and diffraction of light, $7^{\text {th }}$ ed. Cambridge University Press, 1999.

15. A. Turpin, Y. V. Loiko, T. K. Kalkandjiev, and J. Mompart, "Conical refraction: fundamentals and applications", Laser Photonics Rev., 1-22 (2016).

16. M. V. Berry, "Conical diffraction asymptotics: fine structure of Poggendorff rings and axial spike", J. Opt. A: Pure Appl. Opt. 6, 289-230 (2004).

17. D. P. O’Dwyer, C. F. Phelan, Y. P. Rakovich, P. R. Eastham, J. G. Lunney, and J. F. Donegan, "Generation of continuously tunable fractional optical angular momentum using internal conical diffraction", Opt. Express, 18 (16), 16480-16485 (2010).

18. A. Peinado, A.Turpin, C. Iemmi, A. Márquez, T. K. Kalkandjiev, J. Mompart, and J. Campos, "Interferometric characterization of the structured polarized light beam produced by the conical refraction phenomenon", Opt. Express 23, 18080-18091 (2015).

19. M. V. Berry \& S. Klein, "Geometric phases from stacks of crystal plates", Journal of Modern Optics, 43:1, 165-180 (1996).

20. A. M. Belskii and A. P. Khapalyuk, "Internal conical refraction of bounded light beams in biaxial crystals", Opt. Spectrosc. (USSR), 44, 436-439 (1978).

21. M. V. Berry, "Conical diffraction asymptotics: fine structure of Poggendorff rings and axial spike", J. Opt. A: Pure Appl. Opt. 6, 289-230 (2004).

22. A. Brenier, "Lasing with conical diffraction feature in the $\mathrm{KGd}(\mathrm{WO} 4)_{2}: \mathrm{Nd}$ biaxial crystal", Appl. Phys. B: Lasers and Optics, 122, 237 (2016).

23. A. Brenier, A. Majchrowski and E. Michalski, "Light propagation properties of the $\mathrm{Bi}_{2} \mathrm{ZnOB}_{2} \mathrm{O}_{6}$ acentric biaxial crystal: angular orbital momentum from conical diffraction”, Opt. Mat. 91, 286-291 (2019).

24. A. Turpin, Yu. V. Loiko, A. Peinado, A. Lizana, T. K. Kalkandjiev, J. Campos, and J. Mompart, "Polarization tailored novel vector beams based on conical Refraction", Opt. Exp. vol. 23 (5), 5704-5715 (2015).

25. M. V. Berry, M. R. Jeffrey, and M. Mansuripur, "Orbital and spin angular momentum in conical diffraction", J. opt. A: pure Appl. Opt. 7, 685-690 (2005).

26. C. G. Darwin, "Note on the theory of radiation", Proc. R. Soc. Lond., A Contain. Pap. Math. Phys. Character. 136 (829), 36-52 (1932). 
27. S. N. Alperin, R. D. Niederriter, J. T. Gopinath and M. E. Siemens, "Quantitative measurements of the orbital angular momentum of light with a single, stationary lens", Opt. Lett. 41 (21), 5019-5022 (2016).

\section{Figure captions}

1. Set-up for interferentiel conoscopy. After the BS the two beams have been separated for a better visualization by actually they are superimposed.

2. Typical interferograms visualizing Eq. (7), the values in white are $\emptyset_{P}$. a1-a2, b1-b2, c1c2 correspond respectively to the fields of subsections 4.2, 4.3.2 and 4.3.3 .

3. $\theta\left(k_{x}, k_{y}\right)(\mathrm{a} 1)$ : experimental,(a2): theoretical) and $\varphi\left(k_{x}, k_{y}\right)(\mathrm{b} 1)$ : experimental,(b2): theoretical) angular positions of the polarization on the Poincaré sphere.

4. a1, b1, c1: phase of the $\widehat{U}$ operator, $d 1$ : output intensity after CD; $a 2, b 2$ : experimental and theoretical Pancharatman phase difference between the reference beam and the charge 1 beam produced by $\mathrm{CD} ; \mathrm{c} 2, \mathrm{~d} 2$ : experimental and theoretical intensity of the charge 1 beam produced by $\mathrm{CD}$; a3, b3: experimental and theoretical Pancharatman phase difference between the reference beam and the fractional charge beam produced by $\mathrm{CD}$; $\mathrm{c} 3, \mathrm{~d} 3$ : experimental and theoretical intensity of the fractional charge beam produced by $\mathrm{CD}$. 